\title{
Identification of Chrysanthemum Genetic Resources Resistant to White Rust Caused by Puccinia horiana
}

\author{
Sang Kun Park ${ }^{1}$, Jin Hee Lim², Hak Ki Shin ${ }^{1}$, Jae A Jung ${ }^{1}$, Young Soon Kwon', Mi Seon Kim, Ki Sun Kim \\ ${ }^{1}$ National Institute of Horticultural and Herbal Science, Rural Development Administration, Suwon 440-310, Republic of Korea \\ ${ }^{2}$ Plant Engineering Research Institute, Sejong University, Seoul 143-747, Republic of Korea \\ ${ }^{3}$ Department of Plant Science, Seoul National University, Seoul 151-921, Republic of Korea
}

\begin{abstract}
This experiment was carried out to identify genetic resources of chrysanthemum wild species and commercial cultivars that are resistant to white rust disease caused by Puccinia horiana. A total of 190 chrysanthemum accessions, composed of 11 accessions belonging to six wild species and 179 commercial cultivars, were screened for resistance to $P$. horiana isolates collected from Muan and Jeonju, the main production area of chrysanthemum in Korea. A total of 41 spray cultivars and three wild species (Chrysanthemum boreale, C. yoshinaganthum, and C. zawadskii) were identified as resistant. Nine standard and 28 spray cultivars with poorly developed chlorotic lesions and pustules on the leaves were moderate resistant. The remaining 18 standard, 83 spray cultivars and three wild species (Aster spathulifolius, C. indicum, and C. pacificum) were highly susceptible. The use of resistant cultivars is one of the most efficient approaches for overcoming white rust disease in chrysanthemum cultivation. Therefore, these genetic resources can be used in crossbreeding programs for developing white rust resistant chrysanthemum cultivars.
\end{abstract}

Keywords Chrysanthemum, Pathogenicity, Puccinia horiana, Resistant cultivar, Susceptibility, Varietal resistance, White Rust

\section{INTRODUCTION}

Chrysanthemum (Dendranthema x grandiflorum Kitamura), mainly used for cut flowers or as a potted plant, is the second most important ornamental crop, after rose, in Korea. In 2012, it was cultivated on an area of 585 ha; and 270 million stems and 11 million pots were marketed in Korean flower auctions. Moreover, chrysanthemum is one of Korea's main exported floricultural crops. The value of exports, which totaled only 2.1 million dollars in 1999 when the export of the cut flowers of chrysanthemum to Japan began in earnest, hit 10 million dollars in 2012 (MAFRA 2013). Recently, however, as plant quarantine in Japan has been strengthened, volumes of chrysanthemum exports are expected to drop due to the serious infestation of white rust disease in Korea.

Chrysanthemum white rust, caused by Puccinia horiana Henn., is the most destructive fungal disease of chrysanthemum in Korea (Park and Kim 1993). Infection mainly starts at the upper surface of the leaf and causes pale green to yellow spots (up to $4 \mathrm{~mm}$ in diameter) and then forms raised buff or pinkish pustules on the lower surface of the leaf (Baker 1967). Additionally, in extensively infected plants, infection can occur even in the stems, bracts, flower buds, and florets (Dickens 1970). To prevent damages and economic losses, fungicides are regularly sprayed on chrysanthemum plantations before the plants show symptoms of the disease (Dickens 1990; Stapel and Guerrand 2012).

Several cultural methods also have been proposed to control white rust disease in chrysanthemums. Heat treatment of infected plants at $37-40{ }^{\circ} \mathrm{C}$ for $20 \mathrm{~h}$ or the dipping of cuttings in water at $45^{\circ} \mathrm{C}$ for 5 min have been reported to reduce chlorosis and sporulation (Zadoks et al. 1968). Further, the sporidia have been reported to be so sensitive to desiccation at $90 \%$ relative humidity that an exposure to $80 \%$ relative humidity for $5 \mathrm{~min}$ or $90 \%$ relative humidity for $1 \mathrm{~h}$ eradicates $100 \%$ of the sporidia (Firman and Martin 1968). However, practical use of these

Received June 12, 2014; Revised June 14, 2014; Accepted June 16, 2014; Published June 30, 2014

*Comesponding author Sang Kun Park, theodds@korea.kr, Tel: +82-31-290-6145, Fax: +82-31-290-6189 
methods is difficult because heat treatments can cause the death of terminal buds or whole plants and the malformation of plants. Further, control over relative humidity in large greenhouses is also costly. Therefore, the use of resistant cultivars is the best alternative for controlling white rust disease in chrysanthemum cultivation.

Differences in disease resistance against $P$. horiana among chrysanthemum species and cultivars were reported up until the 1980s. Dickens (1968) investigated the susceptibility of various chrysanthemum species, and Martin and Firman (1970) evaluated the level of resistance in 270 chrysanthemum cultivars. Yamaguchi (1981) also screened 250 chrysanthemum cultivars for resistance to white rust. In addition, De Jong and Rademaker (1986) described four types of reactions of the chrysanthemum cultivars to $P$. horiana. They suggested that a single dominant gene controls resistance in hexaploid chrysanthemums based on the segregations for resistance. Recently, Zeng et al. (2013) reported the variation for resistance to white rust in chrysanthemum species. Despite the yearly introduction of newly bred cultivars, there has been no report regarding the evaluation of commercial cultivars resistant to white rust (Pak et al. 2012).

Moreover, in view of the interaction between hosts and parasites, several studies have demonstrated the existence of differential interactions between $P$. horiana isolates and Chrysanthemum species and the highly complex race structure in this pathosystem (Yamaguchi 1981; De Backer et al. 2011). In spite of the economic importance of chrysanthemum in Korea, however, there is little information on the genetic resources resistant to domestic isolates of $P$. horiana. Therefore, this study was carried out to identify white rust resistance in chrysanthemum species and cultivars against local isolates.

\section{MATERIALS AND METHODS}

\section{Plant materials}

A total of 190 chrysanthemum accessions from the National Institute of Horticultural and Herbal Science (NIHHS) were used to screen for resistance against $P$. horiana. These accessions are composed of 11 accessions belonging to six wild species and 179 commercial cultivars.
Cuttings of about $5 \mathrm{~cm}$ in length with one fully expanded leaf were rooted in a 128-plug tray containing a mixture of peat base compost (Sunshine Mix No. 4, SunGro Horticulture, Canada). The rooted cuttings were planted in the experimental greenhouse of the NIHHS in Suwon, with plant distances of $10 \times 10 \mathrm{~cm}$. The greenhouse was maintained with temperatures ranging from $18-25^{\circ} \mathrm{C}$ during daytime and $12-18^{\circ} \mathrm{C}$ during nighttime.

\section{Inoculations}

In preliminary experiments, different inoculation methods such as placing diseased plants with sporulating lesions among the plants (non-quantitative inoculation) and spraying spore suspension over the plants (semi-quantitative inoculation) were tested in different growing stages of chrysanthemum (Zandvoort et al. 1968; Yamaguchi 1981; De Jong and Rademaker 1986; Takatsu et al. 2000; De Backer et al. 2011). According to the results, non-quantitative inoculation method was more easy, stable, and effective to screen the disease resistance against $P$. horiana in chrysanthemum.

To ensure reproducibility, screenings of resistance were conducted three times in 2009 to 2011. In each experiment, each accession was replicated six times and 'Dancer' and 'Puma White' were used as the resistant and the recessive control, respectively. P. horiana isolates were collected from Muan in 2009 and Jeonju in 2010 and 2011, and inoculated in the respective year of collection. In a month after planting, non-quantitative inoculation was performed by placing heavily infected plants with distances of $40 \mathrm{~cm}$ among the test plants. One week after inoculation, all the test plants were sprayed with water and covered with polyethylene plastic film in the evening to preserve a relative humidity close to $100 \%$. And the night temperature was maintained at $>15^{\circ} \mathrm{C}$ during inoculation (Firman and Martin 1968).

\section{Evaluation of resistance}

The test plants were scored three to five weeks after inoculation. On the basis of the development and progression of pustules in the most severely infected leaves, the disease index was divided into six levels: 0 , no visible symptoms; $1,<5$ developed pustules but sporulated limitedly; $2,<20$ developed pustules and sporulated slowly; $3,<50$ developed 
pustules; 4, $<100$ developed pustules; and 5, $>100$ developed pustules. The resistance levels were determined according to the average disease index as follows: 0.0 , no visible symptom, rated as resistant; $0.0-1.0$, few pustules developed slowly and sporulated limitedly, as moderately resistant; and 2.0-5.0, many pustules developed quickly and sporulated abundantly, as susceptible.

Table 1. Classification of the six wild chrysanthemum species based on resistance levels against white rust caused by P. horiana.

\begin{tabular}{|c|c|c|c|}
\hline $\begin{array}{l}\text { Disease } \\
\text { response }\end{array}$ & $\begin{array}{c}\text { No. of } \\
\text { accessions }\end{array}$ & Species & Cultivars or lines \\
\hline \multirow{3}{*}{$\mathrm{R}$} & \multirow{3}{*}{7} & Chrysanthemum boreale & NHCB-1, NHCB-2 \\
\hline & & C. yoshinaganthum & NHCY-1 \\
\hline & & C. zawadskii & NHCZ-1, NHCZ-2, NHCZ-3, NHCZ-4 \\
\hline \multirow{3}{*}{$\mathrm{S}$} & \multirow{3}{*}{4} & Aster spathulifolius & NHAS-1 \\
\hline & & C. indicum & NHCI-1 \\
\hline & & C. pacificum & NHCP-1, NHCP-2 \\
\hline
\end{tabular}

Table 2. Classification of the 179 chrysanthemum cultivars based on resistance level against white rust caused by $P$. horiana.

\begin{tabular}{|c|c|c|c|}
\hline Type & $\begin{array}{l}\text { Disease } \\
\text { response }\end{array}$ & $\begin{array}{c}\text { No. of } \\
\text { accessions }\end{array}$ & Cultivars \\
\hline \multirow[b]{2}{*}{ Standard } & MR & 9 & $\begin{array}{l}\text { Kokka Akafuji, Otomezakura, Otomezakura Orange, Otomezakura Pink, Otomezakura } \\
\text { Yellow, Suishin, Seikonomakoto, Seikonomakoto III, Seinonami }\end{array}$ \\
\hline & S & 18 & $\begin{array}{l}\text { Baekma, Byakko, Iwanohakusen, Jinba, Kiranouma, Kokka Shunko, Seifu, Seiko no } \\
\text { aki, Seikokaike, Seikoshinnen, Seikoumangetu, Seinoisse, Seinokoto, Seinokyoku, } \\
\text { Seiun, Shuhonochikara White, Shuhonochikara Yellow, Yongma }\end{array}$ \\
\hline \multirow[b]{3}{*}{ Spray } & $\mathrm{R}$ & 41 & $\begin{array}{l}\text { Akira Kazaguruma, Albert heijn, Annecy, Ansella, Bacchus, Biarittz Yellow, Crocodile, } \\
\text { Dalma, Dancer, Dark Westland, Dutchy, Eunhasu, Flush, Hambaek, Handsome, Jeanny, } \\
\text { Jerry, Kingfisher, Kingfisher Cherry, Lexy Red, Marikazaguruma, Melody, Merida } \\
\text { Splendid, Morning, Pink Elisa, Quinty, Quinty Pink, Quinty Red, Recharles, Refondo, } \\
\text { Rodis White, Rodis Yellow, Roma, Sei Amelie, Sei Energy, Sei Falcao, Sei Mini, Sei } \\
\text { Sanp, Sei Soul, Taiyo no Koigokoro, Tobago }\end{array}$ \\
\hline & MR & 28 & $\begin{array}{l}\text { Antigua, Arctic Queen, Bacardi, Bennie Jolink, Biarittz Pink, Bongan, Cassa, Champy, } \\
\text { Fly Catcher, Gama, Gibaeg, Hebo, Husky, Hyangro, Ilweol, Marabou, Panama, Penny } \\
\text { Lane, Piaget, Ping Pong White, Ping Pong Yellow, Sei-Rosa, Sei-Soul Yellow Red, } \\
\text { Vesuvio, Vesuvio Yellow, White King, White Marble, Winia }\end{array}$ \\
\hline & $\mathrm{S}$ & 83 & $\begin{array}{l}\text { Accent, Anastasia, Anastasia Green, Ardilo, Argus, Art Yellow, Artist Yellow, Bijux, } \\
\text { Boris Becker Yellow, Bradford, Buffy, Charming Eye, Chopin, Chopin Dark Pink, } \\
\text { Chopin Orange Pink, Chopin White, Coral Marble, Cosmos King, Creado, Dash, } \\
\text { Deliah Cream, Delilah Yellow, Deliwind Yellow, Dinar, Euro White, Euro Yellow, } \\
\text { Feeling Green, Ford, Froggy, Garcia, Golden Kent, Golden PangPang, Green brid, } \\
\text { Hunt, Ibis Lime, Ibis Sunny, Inga, Kinkazaguruma, Kumsu, Lineker Salmon, Marscort, } \\
\text { Mona Lisa, Mona Lisa Pink, Mona Lisa Splendid, Mona Lisa White, Mona Lisa } \\
\text { Yellow, Moonlight, Namba, Nice, Noa, Noeul, Paco, Patra, Peace Green, Pelican, Pink } \\
\text { PangPang, Plaisir d'Amour, Puma Sunny, Puma White, Puma Yellow, Pure Angel, } \\
\text { Relance, Salinas, Samos, Sei Agness, Sei Alps, Sei Elza, Sei Mariah, Sei Monaco, Sei } \\
\text { Night, Sobaek, Stailion, Swan, Text, Tokyo, Topic, Tumaco, Voyager, Weldon Dark, } \\
\text { Wembley, Whitney PangPang, Zembla, Zembla Lime }\end{array}$ \\
\hline
\end{tabular}




\section{RESULTS}

Among the eleven accessions of wild chrysanthemum consisted of six species, four accessions including Aster spathulifolius, Chrysanthemum indicum, and two $C$. pacificum showed high levels of infection. A number of chlorotic lesions and pustules developed on the leaves very quickly. On the other hand, seven accessions including two C. boreale, C. yoshinaganthum, and four C. zawadskii were determined to be resistant and completely free from infection (Table 1, Table S1).

A total of 179 commercial cultivars, consisted of 27 standard and 152 spray types of chrysanthemum, were evaluated for their levels of resistance to white rust. None of the 27 standard chrysanthemums were highly resistant to the disease. 'Kokka Akafuji', 'Otomezakura', 'Otomezakura Orange', 'Otomezakura Pink' and 'Suishin' showed slight necrotic flecks or small pustules visible on the leaves. And

Table S1. List of chrysanthemum wild species and cultivars tested for resistance to Puccinia horiana and their disease response.

\begin{tabular}{|c|c|c|c|c|c|c|c|c|}
\hline Type & Species & $\begin{array}{l}\text { Accession } \\
\text { No. }^{\mathrm{z}}\end{array}$ & $\begin{array}{l}\text { Cultivars or } \\
\text { lines }\end{array}$ & $\begin{array}{c}\text { 1st } \\
\text { inoculation } \\
(2009)^{\mathrm{y}}\end{array}$ & $\begin{array}{c}2 \text { nd } \\
\text { inoculation } \\
(2010)\end{array}$ & $\begin{array}{c}\text { 3rd } \\
\text { inoculation } \\
(2011)\end{array}$ & Total & Response $^{\mathrm{w}}$ \\
\hline \multirow[t]{11}{*}{ Wild type } & Chrysanthemum boreale & IT232531 & NHCB-1 & $0.00 \pm 0.00 \mathrm{x}$ & $0.00 \pm 0.00$ & $0.00 \pm 0.00$ & $0.00 \pm 0.00$ & $\mathrm{R}$ \\
\hline & & IT232532 & NHCB-2 & $0.00 \pm 0.00$ & $0.00 \pm 0.00$ & $0.00 \pm 0.00$ & $0.00 \pm 0.00$ & $\mathrm{R}$ \\
\hline & $\begin{array}{l}\text { Chrysanthemum } \\
\text { yoshinaganthum }\end{array}$ & - & NHCY-1 & $0.00 \pm 0.00$ & $0.00 \pm 0.00$ & $0.00 \pm 0.00$ & $0.00 \pm 0.00$ & $\mathrm{R}$ \\
\hline & Chrysanthemum zawadskii & IT232527 & NHCZ-1 & $0.00 \pm 0.00$ & $0.00 \pm 0.00$ & $0.00 \pm 0.00$ & $0.00 \pm 0.00$ & $\mathrm{R}$ \\
\hline & & IT232528 & NHCZ-2 & $0.00 \pm 0.00$ & $0.00 \pm 0.00$ & $0.00 \pm 0.00$ & $0.00 \pm 0.00$ & $\mathrm{R}$ \\
\hline & & IT232529 & NHCZ-3 & $0.00 \pm 0.00$ & $0.00 \pm 0.00$ & $0.00 \pm 0.00$ & $0.00 \pm 0.00$ & $\mathrm{R}$ \\
\hline & & IT232530 & NHCZ-4 & $0.00 \pm 0.00$ & $0.00 \pm 0.00$ & $0.00 \pm 0.00$ & $0.00 \pm 0.00$ & $\mathrm{R}$ \\
\hline & Aster spathulifolius & - & NHAS-1 & $3.66 \pm 0.51$ & $3.00 \pm 0.89$ & $3.83 \pm 0.98$ & $3.50 \pm 0.85$ & $\mathrm{~S}$ \\
\hline & Chrysanthemum indicum & IT232536 & NHCI-1 & $2.40 \pm 0.54$ & $1.66 \pm 0.81$ & $1.83 \pm 0.98$ & $1.94 \pm 0.92$ & $\mathrm{~S}$ \\
\hline & Chrysanthemum pacificum & IT232538 & NHCP-1 & $4.00 \pm 0.00$ & $3.50 \pm 0.83$ & $4.66 \pm 0.51$ & $4.05 \pm 0.72$ & $\mathrm{~S}$ \\
\hline & & - & NHCP-2 & $3.16 \pm 0.40$ & $3.00 \pm 0.00$ & $4.00 \pm 0.00$ & $3.38 \pm 0.50$ & $\mathrm{~S}$ \\
\hline \multirow[t]{17}{*}{ Standard } & $\begin{array}{l}\text { Dendranthema } \\
\text { grandiflorum }\end{array}$ & IT244120 & Kokka Akafuji & $0.00 \pm 0.00$ & $0.16 \pm 0.40$ & $0.16 \pm 0.40$ & $0.11 \pm 0.32$ & MR \\
\hline & & IT244130 & Otomezakura & $0.16 \pm 0.40$ & $0.16 \pm 0.40$ & $0.00 \pm 0.00$ & $0.11 \pm 0.32$ & MR \\
\hline & & IT244131 & $\begin{array}{l}\text { Otomezakura } \\
\text { Orange }\end{array}$ & $0.00 \pm 0.00$ & $0.00 \pm 0.00$ & $0.16 \pm 0.40$ & $0.05 \pm 0.23$ & MR \\
\hline & & - & $\begin{array}{l}\text { Otomezakura } \\
\text { Pink }\end{array}$ & $0.00 \pm 0.00$ & $0.16 \pm 0.40$ & $0.33 \pm 0.51$ & $0.16 \pm 0.38$ & MR \\
\hline & & IT234194 & $\begin{array}{l}\text { Otomezakura } \\
\text { Yellow }\end{array}$ & $0.16 \pm 0.40$ & $0.20 \pm 0.44$ & $0.16 \pm 0.40$ & $0.17 \pm 0.38$ & MR \\
\hline & & IT234192 & Suishin & $0.16 \pm 0.40$ & $0.33 \pm 0.51$ & $0.16 \pm 0.40$ & $0.22 \pm 0.42$ & MR \\
\hline & & IT245131 & Seikonomakoto & $0.50 \pm 0.54$ & $0.83 \pm 0.75$ & $0.33 \pm 0.51$ & $0.55 \pm 0.61$ & MR \\
\hline & & IT244981 & $\begin{array}{l}\text { Seikonomakoto } \\
\text { III }\end{array}$ & $0.40 \pm 0.54$ & $0.50 \pm 0.54$ & $0.66 \pm 0.51$ & $0.52 \pm 0.51$ & MR \\
\hline & & IT234189 & Seinonami & $0.33 \pm 0.51$ & $0.66 \pm 0.51$ & $0.66 \pm 0.81$ & $0.55 \pm 0.61$ & MR \\
\hline & & IT232551 & Baekma & $3.66 \pm 0.51$ & $3.16 \pm 1.16$ & $4.33 \pm 0.81$ & $3.72 \pm 0.95$ & $\mathrm{~S}$ \\
\hline & & IT234136 & Byakko & $4.66 \pm 0.51$ & $3.00 \pm 0.00$ & $3.16 \pm 0.75$ & $3.61 \pm 0.91$ & $\mathrm{~S}$ \\
\hline & & IT232534 & Iwanohakusen & $2.50 \pm 0.83$ & $2.16 \pm 0.75$ & $2.16 \pm 0.40$ & $2.27 \pm 0.66$ & $\mathrm{~S}$ \\
\hline & & IT232525 & Jinba & $4.83 \pm 0.40$ & $4.60 \pm 0.54$ & $4.50 \pm 0.83$ & $4.64 \pm 1.24$ & $\mathrm{~S}$ \\
\hline & & FCH0203 & Kiranouma & $3.00 \pm 0.00$ & $2.83 \pm 0.40$ & $2.66 \pm 1.50$ & $2.83 \pm 0.85$ & $\mathrm{~S}$ \\
\hline & & - & Kokka Shunko & $5.00 \pm 0.00$ & $5.00 \pm 0.00$ & $5.00 \pm 0.00$ & $5.00 \pm 0.00$ & S \\
\hline & & FCH0110 & Seifu & $5.00 \pm 0.00$ & $5.00 \pm 0.00$ & $5.00 \pm 0.00$ & $5.00 \pm 0.00$ & $\mathrm{~S}$ \\
\hline & & - & Seiko no aki & $5.00 \pm 0.00$ & $5.00 \pm 0.00$ & $5.00 \pm 0.00$ & $5.00 \pm 0.00$ & $\mathrm{~S}$ \\
\hline
\end{tabular}


Table S1. Continued.

\begin{tabular}{|c|c|c|c|c|c|c|c|c|}
\hline Type & Species & $\begin{array}{l}\text { Accession } \\
\text { No. }^{\mathrm{z}}\end{array}$ & $\begin{array}{c}\text { Cultivars or } \\
\text { lines }\end{array}$ & $\begin{array}{c}\text { 1st } \\
\text { inoculation } \\
(2009)^{\mathrm{y}}\end{array}$ & $\begin{array}{c}2 \text { nd } \\
\text { inoculation } \\
(2010)\end{array}$ & $\begin{array}{c}\text { 3rd } \\
\text { inoculation } \\
(2011)\end{array}$ & Total & Response $^{\mathrm{w}}$ \\
\hline & & FCH0194 & Seikokaike & $1.50 \pm 0.54$ & $1.16 \pm 0.40$ & $1.83 \pm 0.75$ & $1.50 \pm 0.61$ & $\mathrm{~S}$ \\
\hline & & FCH0196 & Seikoshinnen & $2.33 \pm 0.51$ & $2.16 \pm 0.40$ & $1.83 \pm 0.75$ & $2.11 \pm 0.58$ & $\mathrm{~S}$ \\
\hline & & FCH0179 & Seikoumangetu & $2.00 \pm 0.00$ & $2.33 \pm 0.51$ & $2.16 \pm 0.75$ & $2.16 \pm 0.51$ & $\mathrm{~S}$ \\
\hline & & IT234188 & Seinoisse & $1.50 \pm 0.83$ & $1.16 \pm 0.40$ & $1.50 \pm 0.54$ & $1.38 \pm 0.60$ & $\mathrm{~S}$ \\
\hline & & FCH0201 & Seinokoto & $3.16 \pm 0.40$ & $3.00 \pm 0.00$ & $2.83 \pm 0.40$ & $3.00 \pm 0.34$ & $\mathrm{~S}$ \\
\hline & & IT234190 & Seinokyoku & $0.83 \pm 0.40$ & $1.50 \pm 0.54$ & $1.00 \pm 0.70$ & $1.11 \pm 0.63$ & $\mathrm{~S}$ \\
\hline & & FCH0197 & Seiun & $4.33 \pm 0.51$ & $4.00 \pm 0.00$ & $3.83 \pm 0.98$ & $4.05 \pm 0.63$ & $\mathrm{~S}$ \\
\hline & & IT244128 & $\begin{array}{l}\text { Shuhonochikara } \\
\text { White }\end{array}$ & $5.00 \pm 0.00$ & $5.00 \pm 0.00$ & $5.00 \pm 0.00$ & $5.00 \pm 0.00$ & $\mathrm{~S}$ \\
\hline & & IT244129 & $\begin{array}{l}\text { Shuhonochikara } \\
\text { Yellow }\end{array}$ & $5.00 \pm 0.00$ & $5.00 \pm 0.00$ & $5.00 \pm 0.00$ & $5.00 \pm 0.00$ & $\mathrm{~S}$ \\
\hline & & IT232548 & Yongma & $4.50 \pm 0.83$ & $3.00 \pm 1.54$ & $4.60 \pm 0.54$ & $4.00 \pm 1.55$ & $\mathrm{~S}$ \\
\hline \multirow[t]{32}{*}{ Spray } & \multirow[t]{32}{*}{$\begin{array}{l}\text { Dendranthema } \\
\text { grandiflorum }\end{array}$} & IT245424 & $\begin{array}{l}\text { Akira } \\
\text { Kazaguruma }\end{array}$ & $0.00 \pm 0.00$ & $0.00 \pm 0.00$ & $0.00 \pm 0.00$ & $0.00 \pm 0.00$ & $\mathrm{R}$ \\
\hline & & FCH0193 & Albert heijn & $0.00 \pm 0.00$ & $0.00 \pm 0.00$ & $0.00 \pm 0.00$ & $0.00 \pm 0.00$ & $\mathrm{R}$ \\
\hline & & $\mathrm{FCH} 0215$ & Annecy & $0.00 \pm 0.00$ & $0.00 \pm 0.00$ & $0.00 \pm 0.00$ & $0.00 \pm 0.00$ & $\mathrm{R}$ \\
\hline & & - & Ansella & $0.00 \pm 0.00$ & $0.00 \pm 0.00$ & $0.00 \pm 0.00$ & $0.00 \pm 0.00$ & $\mathrm{R}$ \\
\hline & & - & Bacchus & $0.00 \pm 0.00$ & $0.00 \pm 0.00$ & $0.00 \pm 0.00$ & $0.00 \pm 0.00$ & $\mathrm{R}$ \\
\hline & & IT244119 & Biarittz Yellow & $0.00 \pm 0.00$ & $0.00 \pm 0.00$ & $0.00 \pm 0.00$ & $0.00 \pm 0.00$ & $\mathrm{R}$ \\
\hline & & - & Crocodile & $0.00 \pm 0.00$ & $0.00 \pm 0.00$ & $0.00 \pm 0.00$ & $0.00 \pm 0.00$ & $\mathrm{R}$ \\
\hline & & IT232543 & Dalma & $0.00 \pm 0.00$ & $0.00 \pm 0.00$ & $0.00 \pm 0.00$ & $0.00 \pm 0.00$ & $\mathrm{R}$ \\
\hline & & IT244123 & Dancer & $0.00 \pm 0.00$ & $0.00 \pm 0.00$ & $0.00 \pm 0.00$ & $0.00 \pm 0.00$ & $\mathrm{R}$ \\
\hline & & - & Dark Westland & $0.00 \pm 0.00$ & $0.00 \pm 0.00$ & $0.00 \pm 0.00$ & $0.00 \pm 0.00$ & $\mathrm{R}$ \\
\hline & & - & Dutchy & $0.00 \pm 0.00$ & $0.00 \pm 0.00$ & $0.00 \pm 0.00$ & $0.00 \pm 0.00$ & $\mathrm{R}$ \\
\hline & & - & Eunhasu & $0.00 \pm 0.00$ & $0.00 \pm 0.00$ & $0.00 \pm 0.00$ & $0.00 \pm 0.00$ & $\mathrm{R}$ \\
\hline & & - & Flush & $0.00 \pm 0.00$ & $0.00 \pm 0.00$ & $0.00 \pm 0.00$ & $0.00 \pm 0.00$ & $\mathrm{R}$ \\
\hline & & IT232553 & Hambaek & $0.00 \pm 0.00$ & $0.00 \pm 0.00$ & $0.00 \pm 0.00$ & $0.00 \pm 0.00$ & $\mathrm{R}$ \\
\hline & & FCH0176 & Handsome & $0.00 \pm 0.00$ & $0.00 \pm 0.00$ & $0.00 \pm 0.00$ & $0.00 \pm 0.00$ & $\mathrm{R}$ \\
\hline & & $\mathrm{FCH} 0140$ & Jeanny & $0.00 \pm 0.00$ & $0.00 \pm 0.00$ & $0.00 \pm 0.00$ & $0.00 \pm 0.00$ & $\mathrm{R}$ \\
\hline & & - & Jerry & $0.00 \pm 0.00$ & $0.00 \pm 0.00$ & $0.00 \pm 0.00$ & $0.00 \pm 0.00$ & $\mathrm{R}$ \\
\hline & & IT245133 & Kingfisher & $0.00 \pm 0.00$ & $0.00 \pm 0.00$ & $0.00 \pm 0.00$ & $0.00 \pm 0.00$ & $\mathrm{R}$ \\
\hline & & - & $\begin{array}{l}\text { Kingfisher } \\
\text { Cherry }\end{array}$ & $0.00 \pm 0.00$ & $0.00 \pm 0.00$ & $0.00 \pm 0.00$ & $0.00 \pm 0.00$ & $\mathrm{R}$ \\
\hline & & - & Lexy Red & $0.00 \pm 0.00$ & $0.00 \pm 0.00$ & $0.00 \pm 0.00$ & $0.00 \pm 0.00$ & $\mathrm{R}$ \\
\hline & & IT245425 & Marikazaguruma & $0.00 \pm 0.00$ & $0.00 \pm 0.00$ & $0.00 \pm 0.00$ & $0.00 \pm 0.00$ & $\mathrm{R}$ \\
\hline & & - & Melody & $0.00 \pm 0.00$ & $0.00 \pm 0.00$ & $0.00 \pm 0.00$ & $0.00 \pm 0.00$ & $\mathrm{R}$ \\
\hline & & - & Merida Splendid & $0.00 \pm 0.00$ & $0.00 \pm 0.00$ & $0.00 \pm 0.00$ & $0.00 \pm 0.00$ & $\mathrm{R}$ \\
\hline & & - & Morning & $0.00 \pm 0.00$ & $0.00 \pm 0.00$ & $0.00 \pm 0.00$ & $0.00 \pm 0.00$ & $\mathrm{R}$ \\
\hline & & - & Pink Elisa & $0.00 \pm 0.00$ & $0.00 \pm 0.00$ & $0.00 \pm 0.00$ & $0.00 \pm 0.00$ & $\mathrm{R}$ \\
\hline & & IT244984 & Quinty & $0.00 \pm 0.00$ & $0.00 \pm 0.00$ & $0.00 \pm 0.00$ & $0.00 \pm 0.00$ & $\mathrm{R}$ \\
\hline & & - & Quinty Pink & $0.00 \pm 0.00$ & $0.00 \pm 0.00$ & $0.00 \pm 0.00$ & $0.00 \pm 0.00$ & $\mathrm{R}$ \\
\hline & & IT245139 & Quinty Red & $0.00 \pm 0.00$ & $0.00 \pm 0.00$ & $0.00 \pm 0.00$ & $0.00 \pm 0.00$ & $\mathrm{R}$ \\
\hline & & - & Recharles & $0.00 \pm 0.00$ & $0.00 \pm 0.00$ & $0.00 \pm 0.00$ & $0.00 \pm 0.00$ & $\mathrm{R}$ \\
\hline & & - & Refondo & $0.00 \pm 0.00$ & $0.00 \pm 0.00$ & $0.00 \pm 0.00$ & $0.00 \pm 0.00$ & $\mathrm{R}$ \\
\hline & & FCH0183 & Rodis White & $0.00 \pm 0.00$ & $0.00 \pm 0.00$ & $0.00 \pm 0.00$ & $0.00 \pm 0.00$ & $\mathrm{R}$ \\
\hline & & FCH0184 & Rodis Yellow & $0.00 \pm 0.00$ & $0.00 \pm 0.00$ & $0.00 \pm 0.00$ & $0.00 \pm 0.00$ & $\mathrm{R}$ \\
\hline
\end{tabular}


Table S1. Continued.

\begin{tabular}{|c|c|c|c|c|c|c|c|c|}
\hline Type & Species & $\begin{array}{l}\text { Accession } \\
\text { No. }^{\mathrm{z}}\end{array}$ & $\begin{array}{c}\text { Cultivars or } \\
\text { lines }\end{array}$ & \multirow{2}{*}{$\begin{array}{c}\text { 1st } \\
\text { inoculation } \\
(2009)^{\mathrm{y}} \\
0.00 \pm 0.00\end{array}$} & \multirow{2}{*}{$\begin{array}{c}\begin{array}{c}\text { nd } \\
\text { inoculation } \\
(2010)\end{array} \\
0.00 \pm 0.00\end{array}$} & \multirow{2}{*}{$\begin{array}{c}\text { 3rd } \\
\text { inoculation } \\
(2011) \\
0.00 \pm 0.00\end{array}$} & \multirow{2}{*}{$\begin{array}{c}\text { Total } \\
0.00 \pm 0.00\end{array}$} & Response $^{w}$ \\
\hline & & FCH0167 & Roma & & & & & $\mathrm{R}$ \\
\hline & & - & Sei Amelie & $0.00 \pm 0.00$ & $0.00 \pm 0.00$ & $0.00 \pm 0.00$ & $0.00 \pm 0.00$ & $\mathrm{R}$ \\
\hline & & FCH0192 & Sei Energy & $0.00 \pm 0.00$ & $0.00 \pm 0.00$ & $0.00 \pm 0.00$ & $0.00 \pm 0.00$ & $\mathrm{R}$ \\
\hline & & - & Sei Falcao & $0.00 \pm 0.00$ & $0.00 \pm 0.00$ & $0.00 \pm 0.00$ & $0.00 \pm 0.00$ & $\mathrm{R}$ \\
\hline & & - & Sei Mini & $0.00 \pm 0.00$ & $0.00 \pm 0.00$ & $0.00 \pm 0.00$ & $0.00 \pm 0.00$ & $\mathrm{R}$ \\
\hline & & - & Sei Sanp & $0.00 \pm 0.00$ & $0.00 \pm 0.00$ & $0.00 \pm 0.00$ & $0.00 \pm 0.00$ & $\mathrm{R}$ \\
\hline & & IT244133 & Sei Soul & $0.00 \pm 0.00$ & $0.00 \pm 0.00$ & $0.00 \pm 0.00$ & $0.00 \pm 0.00$ & $\mathrm{R}$ \\
\hline & & - & $\begin{array}{l}\text { Taiyo no } \\
\text { Koigokoro }\end{array}$ & $0.00 \pm 0.00$ & $0.00 \pm 0.00$ & $0.00 \pm 0.00$ & $0.00 \pm 0.00$ & $\mathrm{R}$ \\
\hline & & - & Tobago & $0.00 \pm 0.00$ & $0.00 \pm 0.00$ & $0.00 \pm 0.00$ & $0.00 \pm 0.00$ & $\mathrm{R}$ \\
\hline & & - & Antigua & $0.50 \pm 0.83$ & $0.50 \pm 0.54$ & $0.66 \pm 0.81$ & $0.55 \pm 0.70$ & MR \\
\hline & & IT245422 & Arctic Queen & $0.40 \pm 0.54$ & $0.83 \pm 0.75$ & $0.50 \pm 0.83$ & $0.58 \pm 0.70$ & MR \\
\hline & & IT234150 & Bacardi & $0.50 \pm 0.83$ & $0.60 \pm 0.89$ & $1.16 \pm 0.98$ & $0.76 \pm 0.89$ & MR \\
\hline & & - & Bennie Jolink & $0.66 \pm 0.81$ & $0.33 \pm 0.51$ & $0.66 \pm 0.51$ & $0.55 \pm 0.61$ & MR \\
\hline & & IT244118 & Biarittz Pink & $0.00 \pm 0.00$ & $0.00 \pm 0.00$ & $0.16 \pm 0.40$ & $0.05 \pm 0.23$ & MR \\
\hline & & - & Bongan & $0.16 \pm 0.40$ & $0.00 \pm 0.00$ & $0.00 \pm 0.00$ & $0.05 \pm 0.23$ & MR \\
\hline & & - & Cassa & $0.16 \pm 0.40$ & $0.00 \pm 0.00$ & $0.16 \pm 0.40$ & $0.11 \pm 0.32$ & MR \\
\hline & & - & Champy & $0.66 \pm 0.51$ & $1.00 \pm 0.00$ & $0.83 \pm 0.40$ & $0.82 \pm 0.42$ & MR \\
\hline & & FCH0175 & Fly Catcher & $0.00 \pm 0.00$ & $0.00 \pm 0.00$ & $0.33 \pm 0.81$ & $0.11 \pm 0.47$ & MR \\
\hline & & IT232559 & Gama & $0.16 \pm 0.40$ & $0.00 \pm 0.00$ & $0.33 \pm 0.51$ & $0.16 \pm 0.38$ & MR \\
\hline & & IT232544 & Gibaeg & $0.00 \pm 0.00$ & $0.00 \pm 0.00$ & $0.16 \pm 0.40$ & $0.05 \pm 0.23$ & MR \\
\hline & & IT232533 & Hebo & $0.66 \pm 0.81$ & $0.50 \pm 0.54$ & $0.50 \pm 0.54$ & $0.55 \pm 0.61$ & MR \\
\hline & & - & Husky & $0.20 \pm 0.44$ & $0.50 \pm 0.83$ & $0.83 \pm 0.75$ & $0.52 \pm 0.70$ & MR \\
\hline & & IT232550 & Hyangro & $0.16 \pm 0.40$ & $0.00 \pm 0.00$ & $0.50 \pm 0.83$ & $0.22 \pm 0.54$ & MR \\
\hline & & IT232555 & Ilweol & $0.50 \pm 0.54$ & $0.33 \pm 0.51$ & $0.83 \pm 0.40$ & $0.55 \pm 0.51$ & MR \\
\hline & & FCH0161 & Marabou & $0.50 \pm 0.54$ & $0.50 \pm 0.54$ & $0.66 \pm 1.03$ & $0.55 \pm 0.70$ & MR \\
\hline & & - & Panama & $0.66 \pm 0.81$ & $0.33 \pm 0.51$ & $0.66 \pm 0.51$ & $0.55 \pm 0.61$ & MR \\
\hline & & IT234103 & Penny Lane & $0.83 \pm 0.98$ & $0.50 \pm 0.83$ & $0.83 \pm 0.40$ & $0.72 \pm 0.75$ & MR \\
\hline & & - & Piaget & $0.50 \pm 0.54$ & $0.40 \pm 0.54$ & $0.83 \pm 0.98$ & $0.58 \pm 0.70$ & MR \\
\hline & & - & $\begin{array}{l}\text { Ping Pong } \\
\text { White }\end{array}$ & $0.33 \pm 0.51$ & $1.00 \pm 0.89$ & $0.33 \pm 0.51$ & $0.55 \pm 0.70$ & MR \\
\hline & & - & $\begin{array}{l}\text { Ping Pong } \\
\text { Yellow }\end{array}$ & $0.50 \pm 0.54$ & $0.60 \pm 0.54$ & $1.00 \pm 0.63$ & $0.70 \pm 0.59$ & MR \\
\hline & & - & Sei-Rosa & $0.50 \pm 0.54$ & $0.33 \pm 0.51$ & $1.00 \pm 0.89$ & $0.61 \pm 0.69$ & MR \\
\hline & & - & $\begin{array}{l}\text { Sei-Soul Yellow } \\
\text { Red }\end{array}$ & $0.20 \pm 0.44$ & $0.00 \pm 0.00$ & $0.66 \pm 0.51$ & $0.29 \pm 0.46$ & MR \\
\hline & & FCH0005 & Vesuvio & $0.16 \pm 0.40$ & $0.00 \pm 0.00$ & $0.66 \pm 0.51$ & $0.27 \pm 0.46$ & MR \\
\hline & & - & Vesuvio Yellow & $0.00 \pm 0.00$ & $0.16 \pm 0.40$ & $0.50 \pm 0.54$ & $0.22 \pm 0.42$ & MR \\
\hline & & - & White King & $0.00 \pm 0.00$ & $0.00 \pm 0.00$ & $0.83 \pm 0.40$ & $0.27 \pm 0.46$ & MR \\
\hline & & - & White Marble & $0.60 \pm 0.54$ & $0.66 \pm 0.81$ & $0.50 \pm 0.83$ & $0.58 \pm 0.70$ & MR \\
\hline & & - & Winia & $0.33 \pm 0.51$ & $0.40 \pm 0.54$ & $0.83 \pm 0.98$ & $0.52 \pm 0.70$ & MR \\
\hline & & - & Accent & $3.66 \pm 0.81$ & $4.66 \pm 0.51$ & $3.83 \pm 0.75$ & $4.05 \pm 0.80$ & $\mathrm{~S}$ \\
\hline & & - & Anastasia & $3.00 \pm 0.63$ & $3.83 \pm 1.47$ & $3.50 \pm 1.76$ & $3.44 \pm 1.33$ & $\mathrm{~S}$ \\
\hline & & IT232558 & Anastasia Green & $4.33 \pm 0.51$ & $4.33 \pm 1.21$ & $4.00 \pm 1.67$ & $4.22 \pm 1.16$ & $\mathrm{~S}$ \\
\hline & & FCH0085 & Ardilo & $2.00 \pm 0.63$ & $1.66 \pm 0.51$ & $2.16 \pm 1.16$ & $1.94 \pm 0.80$ & $\mathrm{~S}$ \\
\hline & & IT234117 & Argus & $3.16 \pm 0.40$ & $4.00 \pm 1.09$ & $3.33 \pm 1.21$ & $3.50 \pm 0.98$ & $\mathrm{~S}$ \\
\hline
\end{tabular}


Table S1. Continued.

\begin{tabular}{|c|c|c|c|c|c|c|c|c|}
\hline Type & Species & $\begin{array}{l}\text { Accession } \\
\text { No. }^{\mathrm{z}}\end{array}$ & $\begin{array}{c}\text { Cultivars or } \\
\text { lines }\end{array}$ & \multirow[t]{2}{*}{$\begin{array}{c}\text { 1st } \\
\text { inoculation } \\
(2009)^{\mathrm{y}} \\
5.00 \pm 0.00\end{array}$} & \multirow[t]{2}{*}{$\begin{array}{c}2 \text { nd } \\
\text { inoculation } \\
(2010) \\
500 \pm 000\end{array}$} & \multirow{2}{*}{$\begin{array}{c}\text { 3rd } \\
\text { inoculation } \\
(2011) \\
5.00 \pm 0.00\end{array}$} & Total & Response $^{\mathrm{w}}$ \\
\hline & & - & Art Yellow & & & & $5.00 \pm 0.00$ & $\mathrm{~S}$ \\
\hline & & - & Artist Yellow & $5.00 \pm 0.00$ & $4.16 \pm 0.40$ & $4.66 \pm 0.51$ & $4.61 \pm 0.50$ & $\mathrm{~S}$ \\
\hline & & FCH0033 & Bijux & $2.66 \pm 0.81$ & $3.16 \pm 0.98$ & $3.00 \pm 0.63$ & $2.94 \pm 0.80$ & $\mathrm{~S}$ \\
\hline & & - & $\begin{array}{l}\text { Boris Becker } \\
\text { Yellow }\end{array}$ & $1.50 \pm 0.83$ & $0.50 \pm 0.83$ & $1.33 \pm 1.21$ & $1.11 \pm 1.02$ & $\mathrm{~S}$ \\
\hline & & IT245421 & Bradford & $4.00 \pm 0.63$ & $3.16 \pm 1.32$ & $5.00 \pm 0.00$ & $4.00 \pm 1.43$ & $\mathrm{~S}$ \\
\hline & & - & Buffy & $3.66 \pm 1.03$ & $4.16 \pm 0.75$ & $3.33 \pm 0.81$ & $3.72 \pm 0.89$ & $\mathrm{~S}$ \\
\hline & & IT232568 & Charming Eye & $2.16 \pm 0.40$ & $1.66 \pm 0.51$ & $2.66 \pm 0.51$ & $2.16 \pm 0.61$ & $\mathrm{~S}$ \\
\hline & & FCH0097 & Chopin & $3.16 \pm 0.40$ & $3.00 \pm 0.00$ & $4.00 \pm 0.00$ & $3.38 \pm 0.50$ & $\mathrm{~S}$ \\
\hline & & FCH0112 & $\begin{array}{l}\text { Chopin Dark } \\
\text { Pink }\end{array}$ & $2.83 \pm 0.40$ & $2.33 \pm 0.51$ & $3.16 \pm 0.40$ & $2.77 \pm 0.54$ & $\mathrm{~S}$ \\
\hline & & - & $\begin{array}{l}\text { Chopin Orange } \\
\text { Pink }\end{array}$ & $2.20 \pm 0.44$ & $2.00 \pm 0.00$ & $3.40 \pm 0.54$ & $2.50 \pm 1.06$ & $\mathrm{~S}$ \\
\hline & & FCH0113 & Chopin White & $4.00 \pm 0.63$ & $2.50 \pm 1.22$ & $4.66 \pm 0.81$ & $3.72 \pm 1.27$ & $\mathrm{~S}$ \\
\hline & & - & Coral Marble & $3.16 \pm 0.40$ & $4.16 \pm 0.40$ & $2.50 \pm 0.54$ & $3.27 \pm 0.82$ & $\mathrm{~S}$ \\
\hline & & - & Cosmos King & $2.16 \pm 0.98$ & $0.50 \pm 0.54$ & $2.80 \pm 0.44$ & $1.76 \pm 1.23$ & $\mathrm{~S}$ \\
\hline & & - & Creado & $3.16 \pm 0.40$ & $3.00 \pm 0.00$ & $3.66 \pm 1.21$ & $3.27 \pm 0.75$ & $\mathrm{~S}$ \\
\hline & & - & Dash & $4.83 \pm 0.40$ & $4.16 \pm 0.40$ & $4.16 \pm 0.40$ & $4.38 \pm 0.50$ & $\mathrm{~S}$ \\
\hline & & FCH0153 & Deliah Cream & $4.00 \pm 0.00$ & $3.16 \pm 0.40$ & $4.00 \pm 0.00$ & $3.72 \pm 0.46$ & $\mathrm{~S}$ \\
\hline & & IT244137 & Delilah Yellow & $3.33 \pm 0.51$ & $1.66 \pm 0.81$ & $3.33 \pm 0.51$ & $2.77 \pm 1.00$ & $\mathrm{~S}$ \\
\hline & & IT245137 & $\begin{array}{l}\text { Deliwind } \\
\text { Yellow }\end{array}$ & $5.00 \pm 0.00$ & $4.83 \pm 0.40$ & $4.66 \pm 0.51$ & $4.83 \pm 0.38$ & $\mathrm{~S}$ \\
\hline & & - & Dinar & $2.33 \pm 0.51$ & $2.00 \pm 0.00$ & $3.00 \pm 0.00$ & $2.44 \pm 0.51$ & $\mathrm{~S}$ \\
\hline & & FCH0114 & Euro White & $1.83 \pm 0.40$ & $0.83 \pm 0.75$ & $1.60 \pm 0.89$ & $1.41 \pm 0.84$ & $\mathrm{~S}$ \\
\hline & & IT234128 & Euro Yellow & $2.00 \pm 0.00$ & $2.00 \pm 0.63$ & $1.83 \pm 0.75$ & $1.94 \pm 0.53$ & $\mathrm{~S}$ \\
\hline & & FCH0094 & Feeling Green & $5.00 \pm 0.00$ & $4.80 \pm 0.44$ & $5.00 \pm 0.00$ & $4.94 \pm 1.18$ & $\mathrm{~S}$ \\
\hline & & IT244138 & Ford & $5.00 \pm 0.00$ & $5.00 \pm 0.00$ & $5.00 \pm 0.00$ & $5.00 \pm 0.00$ & $\mathrm{~S}$ \\
\hline & & IT245135 & Froggy & $5.00 \pm 0.00$ & $5.00 \pm 0.00$ & $5.00 \pm 0.00$ & $5.00 \pm 0.00$ & $\mathrm{~S}$ \\
\hline & & FCH0096 & Garcia & $2.33 \pm 0.51$ & $1.33 \pm 0.51$ & $2.16 \pm 0.40$ & $1.94 \pm 0.63$ & $\mathrm{~S}$ \\
\hline & & - & Golden Kent & $1.50 \pm 0.83$ & $2.40 \pm 1.51$ & $3.66 \pm 0.81$ & $2.52 \pm 1.46$ & $\mathrm{~S}$ \\
\hline & & IT246016 & $\begin{array}{l}\text { Golden } \\
\text { PangPang }\end{array}$ & $2.60 \pm 0.54$ & $3.33 \pm 1.63$ & $4.33 \pm 1.03$ & $3.47 \pm 1.52$ & $\mathrm{~S}$ \\
\hline & & IT234161 & Green brid & $2.00 \pm 0.00$ & $2.66 \pm 0.51$ & $2.83 \pm 0.75$ & $2.50 \pm 0.61$ & $\mathrm{~S}$ \\
\hline & & - & Hunt & $5.00 \pm 0.00$ & $5.00 \pm 0.00$ & $5.00 \pm 0.00$ & $5.00 \pm 0.00$ & $\mathrm{~S}$ \\
\hline & & - & Ibis Lime & $3.00 \pm 0.00$ & $1.83 \pm 0.40$ & $2.00 \pm 0.00$ & $2.27 \pm 0.57$ & $\mathrm{~S}$ \\
\hline & & FCH0204 & Ibis Sunny & $2.66 \pm 0.51$ & $3.16 \pm 0.40$ & $2.83 \pm 0.40$ & $2.88 \pm 0.47$ & $\mathrm{~S}$ \\
\hline & & IT234180 & Inga & $2.80 \pm 0.44$ & $1.83 \pm 1.16$ & $3.16 \pm 1.60$ & $2.58 \pm 1.38$ & $\mathrm{~S}$ \\
\hline & & IT244122 & Kinkazaguruma & $1.20 \pm 0.44$ & $1.50 \pm 1.04$ & $1.33 \pm 0.51$ & $1.35 \pm 0.75$ & $\mathrm{~S}$ \\
\hline & & IT232542 & Kumsu & $4.16 \pm 0.40$ & $3.16 \pm 0.75$ & $5.00 \pm 0.00$ & $4.11 \pm 0.90$ & $\mathrm{~S}$ \\
\hline & & - & Lineker Salmon & $2.16 \pm 0.75$ & $2.16 \pm 1.47$ & $1.50 \pm 1.37$ & $1.94 \pm 1.21$ & $\mathrm{~S}$ \\
\hline & & - & Marscort & $2.00 \pm 0.00$ & $1.83 \pm 0.40$ & $2.33 \pm 1.21$ & $2.05 \pm 0.72$ & $\mathrm{~S}$ \\
\hline & & - & Mona Lisa & $3.50 \pm 1.04$ & $5.00 \pm 0.00$ & $5.00 \pm 0.00$ & $4.50 \pm 0.92$ & $\mathrm{~S}$ \\
\hline & & - & Mona Lisa Pink & $2.50 \pm 1.22$ & $1.00 \pm 0.63$ & $1.66 \pm 0.81$ & $1.72 \pm 1.07$ & $\mathrm{~S}$ \\
\hline & & IT245138 & $\begin{array}{l}\text { Mona Lisa } \\
\text { Splendid }\end{array}$ & $2.16 \pm 0.40$ & $1.83 \pm 0.40$ & $2.00 \pm 0.00$ & $2.00 \pm 0.34$ & $\mathrm{~S}$ \\
\hline & & IT234168 & $\begin{array}{l}\text { Mona Lisa } \\
\text { White }\end{array}$ & $1.83 \pm 0.75$ & $0.50 \pm 0.54$ & $1.20 \pm 0.83$ & $1.17 \pm 0.90$ & $\mathrm{~S}$ \\
\hline
\end{tabular}


Table S1. Continued.

\begin{tabular}{|c|c|c|c|c|c|c|c|c|}
\hline Type & Species & $\begin{array}{c}\text { Accession } \\
\text { No. }^{\mathrm{z}}\end{array}$ & $\begin{array}{c}\text { Cultivars or } \\
\text { lines }\end{array}$ & $\begin{array}{c}\text { 1st } \\
\text { inoculation } \\
(2009)^{\mathrm{y}}\end{array}$ & $\begin{array}{c}\text { nd } \\
\text { inoculation } \\
(2010)\end{array}$ & $\begin{array}{c}\text { 3rd } \\
\text { inoculation } \\
(2011)\end{array}$ & Total & Response $^{\mathrm{n}}$ \\
\hline & & IT234166 & $\begin{array}{l}\text { Mona Lisa } \\
\text { Yellow }\end{array}$ & $2.00 \pm 0.70$ & $0.60 \pm 0.54$ & $1.50 \pm 0.83$ & $1.37 \pm 0.94$ & $\mathrm{~S}$ \\
\hline & & IT244132 & Moonlight & $3.33 \pm 0.51$ & $2.33 \pm 0.81$ & $2.40 \pm 0.89$ & $2.70 \pm 1.04$ & $\mathrm{~S}$ \\
\hline & & FCH0159 & Namba & $2.33 \pm 0.51$ & $1.83 \pm 0.40$ & $2.66 \pm 0.81$ & $2.27 \pm 0.66$ & $\mathrm{~S}$ \\
\hline & & - & Nice & $4.66 \pm 0.51$ & $4.83 \pm 0.40$ & $5.00 \pm 0.00$ & $4.83 \pm 0.38$ & $\mathrm{~S}$ \\
\hline & & IT234119 & Noa & $4.16 \pm 0.40$ & $5.00 \pm 0.00$ & $4.16 \pm 0.75$ & $4.44 \pm 0.61$ & $\mathrm{~S}$ \\
\hline & & IT232541 & Noeul & $1.16 \pm 0.40$ & $1.40 \pm 0.54$ & $1.20 \pm 0.83$ & $1.25 \pm 0.67$ & $\mathrm{~S}$ \\
\hline & & - & Paco & $1.66 \pm 1.75$ & $0.33 \pm 0.51$ & $1.33 \pm 0.81$ & $1.11 \pm 1.23$ & $\mathrm{~S}$ \\
\hline & & - & Patra & $3.00 \pm 0.00$ & $2.00 \pm 1.26$ & $2.40 \pm 0.89$ & $2.47 \pm 1.08$ & $\mathrm{~S}$ \\
\hline & & - & Peace Green & $4.00 \pm 1.26$ & $2.16 \pm 0.40$ & $4.00 \pm 0.89$ & $3.38 \pm 1.24$ & $\mathrm{~S}$ \\
\hline & & - & Pelican & $1.83 \pm 0.75$ & $1.00 \pm 0.89$ & $2.00 \pm 0.00$ & $1.61 \pm 0.77$ & $\mathrm{~S}$ \\
\hline & & IT232545 & Pink PangPang & $2.83 \pm 0.40$ & $1.40 \pm 0.89$ & $2.00 \pm 0.00$ & $2.11 \pm 0.78$ & $\mathrm{~S}$ \\
\hline & & IT232565 & Plaisir d'Amour & $3.00 \pm 0.00$ & $2.16 \pm 0.40$ & $3.00 \pm 0.00$ & $2.72 \pm 0.46$ & $\mathrm{~S}$ \\
\hline & & FCH0086 & Puma Sunny & $5.00 \pm 0.00$ & $5.00 \pm 0.00$ & $5.00 \pm 0.00$ & $5.00 \pm 0.00$ & $\mathrm{~S}$ \\
\hline & & IT234093 & Puma White & $3.83 \pm 0.40$ & $3.50 \pm 1.22$ & $4.50 \pm 1.22$ & $3.94 \pm 1.05$ & $\mathrm{~S}$ \\
\hline & & FCH0045 & Puma Yellow & $4.83 \pm 0.40$ & $5.00 \pm 0.00$ & $5.00 \pm 0.00$ & $4.94 \pm 0.23$ & $\mathrm{~S}$ \\
\hline & & IT232575 & Pure Angel & $5.00 \pm 0.00$ & $5.00 \pm 0.00$ & $5.00 \pm 0.00$ & $5.00 \pm 0.00$ & S \\
\hline & & IT245134 & Relance & $3.00 \pm 0.00$ & $3.00 \pm 0.00$ & $3.00 \pm 0.00$ & $3.00 \pm 0.00$ & $\mathrm{~S}$ \\
\hline & & FCH0171 & Salinas & $5.00 \pm 0.00$ & $4.00 \pm 1.54$ & $4.33 \pm 1.21$ & $4.44 \pm 1.14$ & $\mathrm{~S}$ \\
\hline & & - & Samos & $2.33 \pm 0.51$ & $2.16 \pm 0.40$ & $2.16 \pm 1.16$ & $2.22 \pm 0.73$ & $\mathrm{~S}$ \\
\hline & & FCH0187 & Sei Agness & $2.16 \pm 0.40$ & $2.16 \pm 1.16$ & $3.33 \pm 1.75$ & $2.55 \pm 1.29$ & $\mathrm{~S}$ \\
\hline & & - & Sei Alps & $3.50 \pm 0.54$ & $3.33 \pm 0.81$ & $3.33 \pm 0.81$ & $3.38 \pm 0.69$ & $\mathrm{~S}$ \\
\hline & & FCH0189 & Sei Elza & $5.00 \pm 0.00$ & $5.00 \pm 0.00$ & $5.00 \pm 0.00$ & $5.00 \pm 1.17$ & $\mathrm{~S}$ \\
\hline & & - & Sei Mariah & $5.00 \pm 0.00$ & $5.00 \pm 0.00$ & $5.00 \pm 0.00$ & $5.00 \pm 0.00$ & $\mathrm{~S}$ \\
\hline & & - & Sei Monaco & $2.33 \pm 1.03$ & $2.83 \pm 0.98$ & $2.33 \pm 1.50$ & $2.50 \pm 1.15$ & $\mathrm{~S}$ \\
\hline & & FCH0186 & Sei Night & $4.00 \pm 1.54$ & $4.66 \pm 0.81$ & $4.50 \pm 1.22$ & $4.38 \pm 1.19$ & $\mathrm{~S}$ \\
\hline & & IT232549 & Sobaek & $2.00 \pm 0.00$ & $2.66 \pm 0.81$ & $2.16 \pm 1.94$ & $2.27 \pm 1.17$ & $\mathrm{~S}$ \\
\hline & & FCH0060 & Stailion & $3.16 \pm 0.40$ & $2.50 \pm 0.54$ & $3.00 \pm 0.00$ & $2.88 \pm 0.47$ & $\mathrm{~S}$ \\
\hline & & IT234172 & Swan & $4.83 \pm 0.40$ & $5.00 \pm 0.00$ & $4.33 \pm 0.81$ & $4.72 \pm 0.57$ & $\mathrm{~S}$ \\
\hline & & FCH0205 & Text & $1.83 \pm 0.98$ & $2.00 \pm 0.63$ & $1.16 \pm 1.32$ & $1.66 \pm 1.02$ & $\mathrm{~S}$ \\
\hline & & - & Tokyo & $1.83 \pm 0.75$ & $4.66 \pm 0.51$ & $2.66 \pm 1.36$ & $3.05 \pm 1.51$ & $\mathrm{~S}$ \\
\hline & & - & Topic & $3.00 \pm 0.00$ & $2.83 \pm 0.40$ & $3.33 \pm 1.03$ & $3.05 \pm 0.63$ & $\mathrm{~S}$ \\
\hline & & IT234181 & Tumaco & $0.16 \pm 0.40$ & $4.83 \pm 0.40$ & $1.66 \pm 0.81$ & $2.22 \pm 2.07$ & $\mathrm{~S}$ \\
\hline & & FCH0185 & Voyager & $3.83 \pm 0.98$ & $4.00 \pm 0.00$ & $5.00 \pm 0.00$ & $4.27 \pm 0.75$ & $\mathrm{~S}$ \\
\hline & & - & Weldon Dark & $1.33 \pm 0.51$ & $1.16 \pm 0.40$ & $2.00 \pm 0.00$ & $1.50 \pm 0.51$ & $\mathrm{~S}$ \\
\hline & & - & Wembley & $5.00 \pm 0.00$ & $4.80 \pm 0.44$ & $5.00 \pm 0.00$ & $4.94 \pm 1.18$ & $\mathrm{~S}$ \\
\hline & & IT232564 & $\begin{array}{l}\text { Whitney } \\
\text { PangPang }\end{array}$ & $1.83 \pm 0.40$ & $3.16 \pm 0.98$ & $0.66 \pm 0.81$ & $1.88 \pm 1.27$ & $\mathrm{~S}$ \\
\hline & & - & Zembla & $0.83 \pm 0.40$ & $1.83 \pm 0.75$ & $2.00 \pm 1.26$ & $1.55 \pm 0.98$ & $\mathrm{~S}$ \\
\hline & & - & Zembla Lime & $1.00 \pm 0.00$ & $1.00 \pm 0.89$ & $1.66 \pm 1.03$ & $1.22 \pm 0.80$ & $\mathrm{~S}$ \\
\hline
\end{tabular}

${ }^{\mathrm{z}}$ Registration number in Rural Development Administration (RDA) Gene Bank Center in Korea.

${ }^{\mathrm{y}}$ The year when inoculation was performed.

${ }^{\mathrm{x}} \mathrm{Mean} \pm \mathrm{SD}$

${ }^{\mathrm{w}}$ The response was determined according to the average disease index as follows: 0.0 , resistant (R); $0.0^{-1.0,}$ moderately resistant (MR); 2.0-5.0, susceptible (S). 
'Seikonomakoto', 'Seikonomakoto III' and 'Seinonami' showed few chlorotic lesions and pustules on the leaves, which developed slowly and sporulated limitedly. These nine cultivars were thus considered moderately resistant. But the other 17 cultivars including 'Baekma', 'Bayakko', 'Iwanohakusen', and 'Jinba' which are widely cultivated in Korea were susceptible as many pustules developed on the leaves very quickly and sporulated abundantly (Table 2, Table S1).

Compared to the standard type, the levels of resistance appeared to be higher in the spray type. Among the 152 spray chrysanthemums, 41 cultivars, such as 'Akira Kazaguruma', 'Biarittz Yellow, 'Bongan', 'Kingfisher', 'Dancer', 'Quinty', among others, were resistant to white rust. Additionally, 28 cultivars, including 'Antigua', 'Bacardi', 'Husky', 'Panama', and 'Sei Soul Yellow Red', among others, exhibited low levels of infection to white rust and were considered moderately resistant. The remaining 83 cultivars were easily infected with white rust, hence considered as susceptible. The infected leaves of these cultivars were quickly covered with numerous pustules. Moreover, in extensively infected cultivars, pustules formed even on the stems, bracts, and flower buds (Table 2, Table S1).

\section{DISCUSSION}

Chrysanthemum white rust, caused by Puccinia horiana Henn., is the most destructive fungal disease in chrysanthemum causing serious production losses. Greenhouse cultivation of this ornamental crop during summer is threatened by the spores build up on the greenhouse curtain wall used for the short day treatment.

Variation for resistance to white rust in various Chrysanthemum species and the differential interactions between P. horiana isolates and Chrysanthemum species have been investigated. However, so far, there is no report available on the genetic resources resistant to local isolates of $P$. horiana. In this study, white rust resistance of wild chrysanthemum species and commercial cultivars against local isolates was studied.

Result of the disease screening test with six wild chrysanthemum species, was consistent with the previous reports (Dikens 1968; Yamaguchi 1981) except for the four accessions of $C$. zawadskii which were resistant in this study but were previously identified as susceptible by Yamaguchi (1981), particularly the C. zawadskii spp. latilobum. The inconsistency between the results for $C$. zawadskii may be due to varietal variations. Most of the cultivated chrysanthemums have been identified predominantly as hexaploid $(2 \mathrm{n}=6 \mathrm{x}=54$ in which the somatic chromosome numbers vary $(2 n=47-63)$ (Dorwick 1952). However, in wild species of chrysanthemum, the polyploidy level varies within the same species. All the four accessions of $C$. zawadskii tested in this experiment and the C. zawadskii spp. latilobum were reported as hexaploid and tetraploid, respectively. Genetic variations in diploid wild species of chrysanthemum could be helpful in analyzing the mode of inheritance for white rust resistance and in detecting resistance genes.

In case of commercial cultivars, among the 179 chrysanthemum cultivars tested in these experiments, 41 spray types were resistant and nine standard cultivars were moderately resistant. The high frequency of resistance to white rust indicated that the resistance, reported to be controlled by a single dominant gene, might have been stably inherited by the next generation (De Jong and Rademaker 1986). Therefore, these genetic resources are expected to be helpful for crossbreeding programs to breed new cultivars resistant to white rust disease in chrysanthemum.

However, differential interactions between $P$. horiana isolates and chrysanthemum cultivars, which have been proposed recently, indicate that more than two types of white rust resistance gene might exist in chrysanthemums (Yamaguchi 1981; De Backer et al. 2011). In this study, two kinds of isolates collected from Muan and Jeonju were inoculated, but interestingly pathogenicity or virulence did not significantly vary (Table S1). Further investigation using the subset of chrysanthemum cultivars identified in this study for disease evaluation using various isolates of $P$. horiana from different location in the main production area should provide us substantial data on suitable cultivar for a specific area. Likewise, these data are valuable to identify resistance genes appropriate for a particular pathotype, and pyramid those genes for improving white rust resistance in chrysanthemums. 


\section{ACKNOWLEDGMENT}

This work was supported by a grant (Code \# PJ008509) from National Institute of Horticultural and Herbal Science, Rural Development Administration, Republic of Korea.

\section{REFERENCES}

Baker JJ. 1967. Chrysanthemum white rust in England and Wales 1963-66. Plant Pathol. 16: 162-166.

De Backer M, Alaei H, Van Bockstaele E, Roldan-Ruiz I, van der Lee T, Maes M, Heungens K. 2011. Identification and characterization of pathotypes in Puccinia horiana, a rust pathogen of Chrysanthemum x morifolium. Eur. J. Plant Pathol. 130: 325-338.

De Jong J, Rademaker W. 1986. The reaction of chrysanthemum cultivars to Puccinia horiana and the inheritance of resistance. Euphytica 35: 945-952.

Dickens JSW. 1968. The resistance of various cultivars and species of chrysanthemum to white rust (Puccinia horiana Henn.). Plant Pathol. 17: 19-22.

Dickens JSW. 1970. Infection of chrysanthemum flowers by white rust (Puccinia horiana Henn.). Plant Pathol. 19: 122-124.

Dickens JSW. 1990. Studies on chemical control of chrysanthemum white rust caused by Puccinia horiana. Plant Pathol. 39: 434-442.

Dorwick GJ. 1952. The chromosome of chrysanthemum, I: The species. Heredity 6: 365-375.

Firman ID, Martin PH. 1968. White rust of chrysanthemum. Ann. Appl. Biol. 62: 429-442.

Martin PH, Firman ID. 1970. Resistance of chrysanthemum cultivars to white rust (Puccinia horiana). Plant Pathol. 19: $180-184$.

Ministry of Agriculture, Food and Rural Affairs (MAFRA). 2013. Annual report of floriculture in 2012. MAFRA, Sejong. p.13-14.

Pak HS, Choi TY, Won MK, Choi WC, Kim DC, Choi HG, Choi BJ. 2012. A new spray chrysanthemum cultivar 'Yes Morning' with single type and puple/white color petal. Kor. J. Breed. Sci. 44: 62-65.

Park KS, Kim CH. 1993. Effect of Temperature and pH on Sporidia formation of Puccinia horiana on chrysanthemum and evaluation of varietal resistance. Kor. J. Plant Pathol. 9: 42-46.

Stapel O, Guerrand J. 2012. High efficacy of possible plant elicitor and other phytostimulants against white rust (Puccinia horiana Henn.) in potted chrysanthemum productions. Acta Hort. (ISHS) 937: 355-359.

Takatsu T, Ohishi K, Tomita Y, Hayashi M, Nakajima M, Akutsu K. 2000. Use of chrysanthemum plantlets grown in vitro to test cultivar susceptibility to white rust, Puccinia horiana P. Hennings. Plant Breed. 119: 528-530.

Yamaguchi T. 1981. Chrysanthemum breeding for resistance to white rust. Japan. J. Breed. 31: 121-132.

Zadoks JC, Groenewegen CAM, Zandvoort R. 1968. On heat treatment of Chrysanthemum morilifolium against Puccinia horiana. Neth. J. Plant Pathol. 74: 25-27.

Zandvoort R, Groenewegen CAM, Zadoks JC. 1968. Methods for the inoculation of Chrysanthemum morifolium with Puccinia horiana. Neth. J. Plant Pathol. 74: 174-176.

Zeng J, Sun J, Xu Y, Chen F, Jiang J, Fang W, Chen S. 2013. Variation for resistance to white rust (Puccinia horiana) among Ajania and Chrysanthemum Species. HortScience 48: 1231-1234. 